\title{
Our Impact Factor 2015 is increased to 1.783: a comment from the Editor-in-Chief
}

\author{
Antonio Federico ${ }^{1}$
}

Published online: 15 July 2016

(C) Springer-Verlag Italia 2016

The Journal Citation Report 2015 has been recently published by Thomson Reuters-ISI, with the impact factor (IF) (2014) derived by the article citations in International Journals on 2013 and 2014.

With satisfaction, we have noted that the Neurological Journal IF, that in the past year was 1.447 , is increased to 1.783 , due to an increased number of citations of more than 200.

This increase is related to the quality of the articles we have published due to the higher selection (we have a rejection rate of more than $70 \%$ ).

With all the well-known limitations surrounding the IF, it remains as a useful measure of the interest provoked by the published articles, and it is one of the many correlations with journal quality to be considered when we submit a paper. In consequence of this, the increase of the IF is usually correlated with a higher possibility to attract good articles.
For all these reasons, we hope to further improve it in the future, with a higher quality original articles and a higher number of good reviews in all fields of neurology.

In this occasion, I wish to thank the editorial board, associated editors and reviewers as well all contributors that considered our journal for publishing their work, hoping that Neurological Sciences with all our efforts will become more and more attractive in the future.

Prof. Antonio Federico

Editor in chief

Neurological Sciences

Compliance with ethical standards

Conflict of interest No conflict of interest.

Antonio Federico

federico@unisi.it

1 Department of Medicine, Surgery and Neurosciences, University of Siena, Siena, Italy 